\title{
Cognitive Ergonomics in Virtual Environments: Development of an Intuitive and Appropriate Input Device for Navigating in a Virtual Maze
}

\author{
Oliver Stefani, ${ }^{1,6}$ Ralph Mager, ${ }^{1}$ Franz Mueller-Spahn, ${ }^{1}$ \\ Hubert Sulzenbacher, ${ }^{2}$ Evangelos Bekiaris, ${ }^{3}$ Brenda K. Wiederhold, ${ }^{4}$ \\ Harshada Patel, ${ }^{5}$ and Alex H. Bullinger ${ }^{1}$
}

\begin{abstract}
For patients suffering from mild cognitive impairments, the navigation through a virtual maze should be as intuitive and efficient as possible in order to minimize cognitive and physical strain. This paper discusses the appropriateness of interaction devices for being used for easy navigation tasks. Information gained from human centered evaluation was used to develop an intuitive and ergonomic interaction device. Two experiments examined the usability of tracked interaction devices. Usability problems with the devices are discussed. The findings from the experiments were translated into general design guidance, in addition to specific recommendations. A new device was designed on the basis of these recommendations and its usability was evaluated in a second experiment. The results were used to develop a lightweight interaction device for navigation in the virtual maze.
\end{abstract}

KEY WORDS: mild cognitive impairment; virtual reality; virtual maze.

\section{INTRODUCTION}

The Virtual Maze (see Fig. 1) is a fully immersive stereoscopic Virtual Environment (VE) for patients suffering from mild cognitive impairments. Entities that can be tested within this VE resemble the classic Wisconsin Card Sorting Test WCST, (Nelson, 1976).

Graphical cues in the form of 3D-objects are given within the virtual maze. The objects are of different colors, different shapes, and of different dynamics (rotating, not moving at all, wobbling). By finding the "right" cue property and following it, the user will find

\footnotetext{
${ }^{1}$ COAT-Basel/UPKBS, Department of Psychiatry, University of Basel, Switzerland.

${ }^{2}$ Psychiatric Clinic, University of Innsbruck, Innsbruck, Austria.

${ }^{3}$ CERTH/HIT, Centre for Research \& Technology Hellas, Athens, Greece.

${ }^{4}$ Virtual Reality Medical Center, San Diego, California.

${ }^{5}$ VIRART, University of Nottingham, Nottingham, UK.

${ }^{6}$ Address all correspondence to Oliver Stefani, COAT-Basel/UPKBS, Center of Applied Technologies in Neuroscience, Wilhelm Klein-Strasse 27, 4025 Basel, Switzerland; e-mail: ols@coat-basel.com.
} 


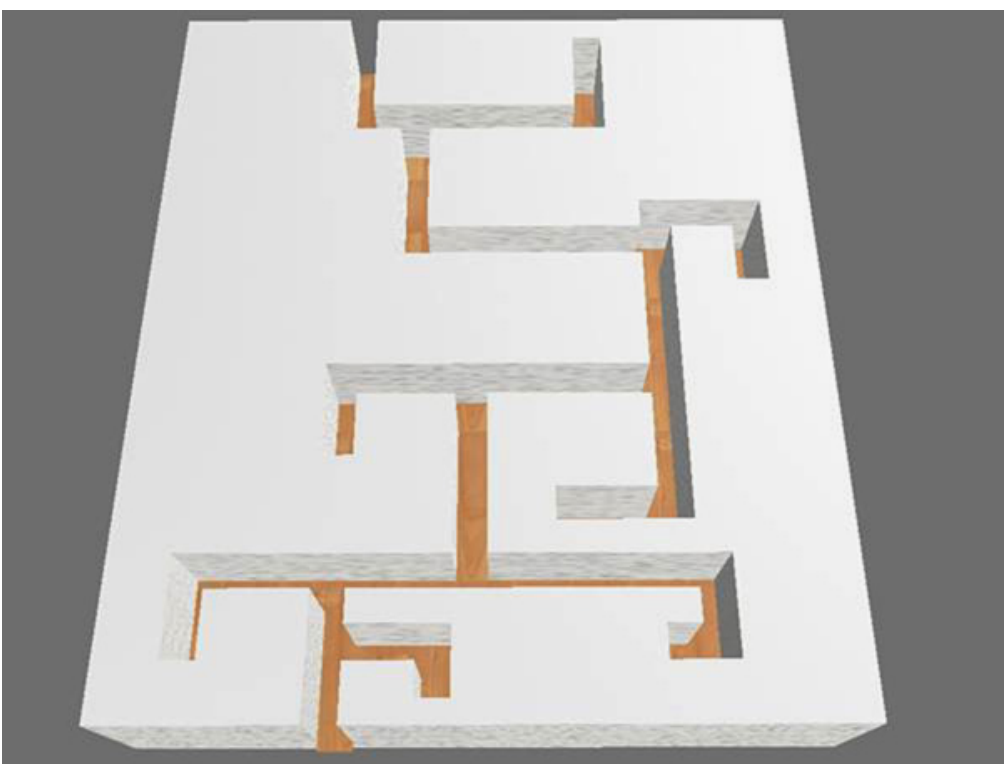

Fig. 1. Snapshot of the virtual maze.

her/his way out of the Virtual Maze. (Software used: Unix and Lightning; Hardware used: SGI Onyx2, V8 HMD as shown in Fig. 2, Ascension Flock of Birds).

The problem was that there are no intuitive but only intricate input devices existing.

Desktop bound input devices like the standard desktop mouse, joysticks, the space mouse (see Fig. 3), or the FlyBox (see Fig. 4) can be used to navigate within a virtual environment but, although they can provide up to six degrees of freedom (DOF), the interaction is not direct and immediate but indirect and oblique (Roessler, 2001).

Additionally, they require a space like a desktop to be placed on. For navigating through the virtual maze those desktop bound input devices decreased the performance of the users drastically. Results obtained from classic WCST were much better than those obtained within the VE. Presently there are many input devices for immersive systems like datagloves, metaphoric input devices (e.g., cubic mouse as shown in Fig. 5 and props), and spatial pointing devices with 6 DOF like the MIKE (Häfner, 2002) all of which are

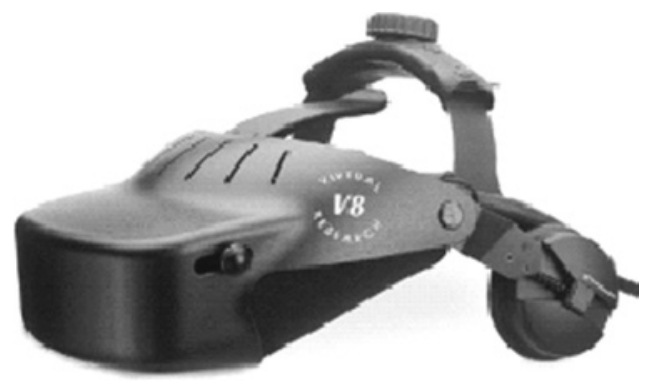

Fig. 2. The V8 HMD. 


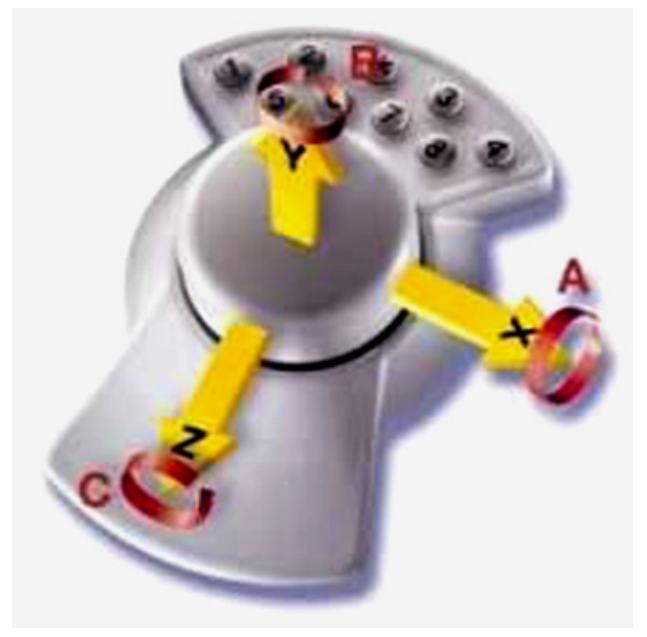

Fig. 3. The space mouse.

optimized for different tracking systems (optical, ultrasonic, electromagnetic, etc.) and different interaction-techniques.

For the simple task of just navigating through a virtual maze those devices are far too complex. Even a wireless device with buttons has shown usability problems (see Fig. 6) because of its heavy weight and the small buttons.

To keep cognitive and physical strain as low as possible, a simple, lightweight device, which could be held in an ergonomic way, was needed. There was no need for buttons or similar because the "go-go" technique (Poupyrev, Billinghurst, Weghorst, \& Ichikawa, 1996) which is similar to the "point and fly" technique (Mine, 1995) was used for navigation. Holding the device close to the body resulted in no movement. Pointing away from the body resulted in a relative acceleration in the pointed direction.

All existing input devices mentioned above, including datagloves are certainly suitable for specific tasks but all of them require intensive training to get used to them. Especially datagloves are not as intuitive to use as one might think. The problem to be solved was the

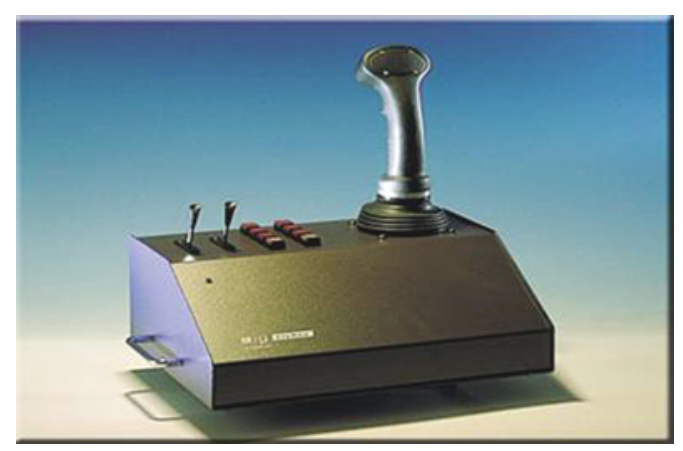

Fig. 4. The flybox. 


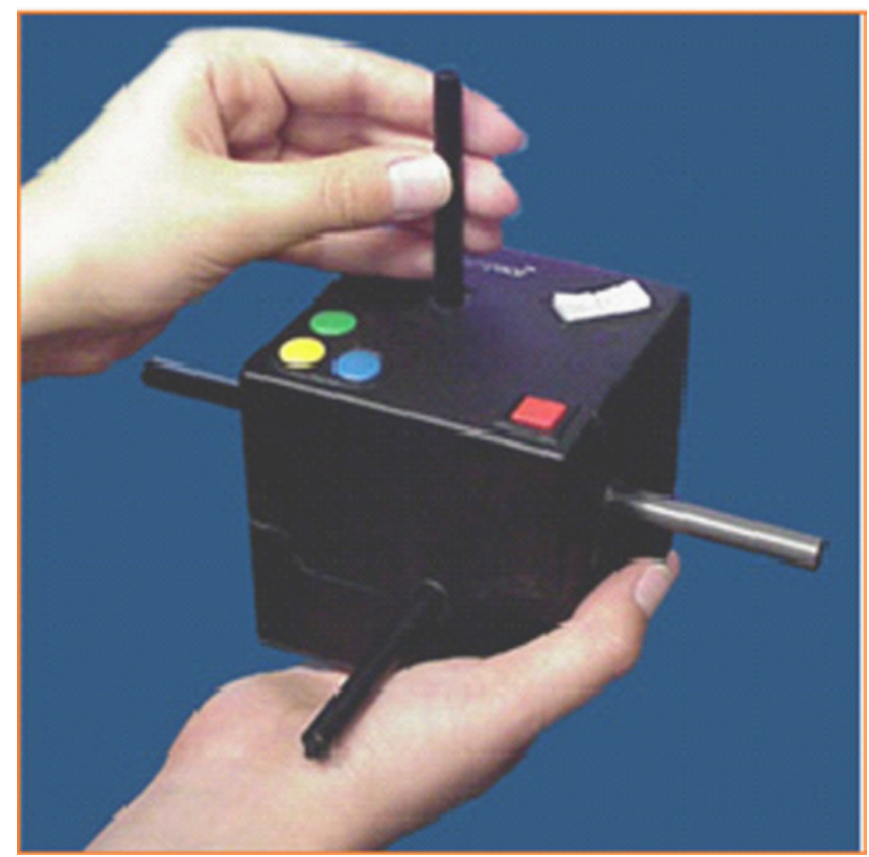

Fig. 5. The cubic mouse.

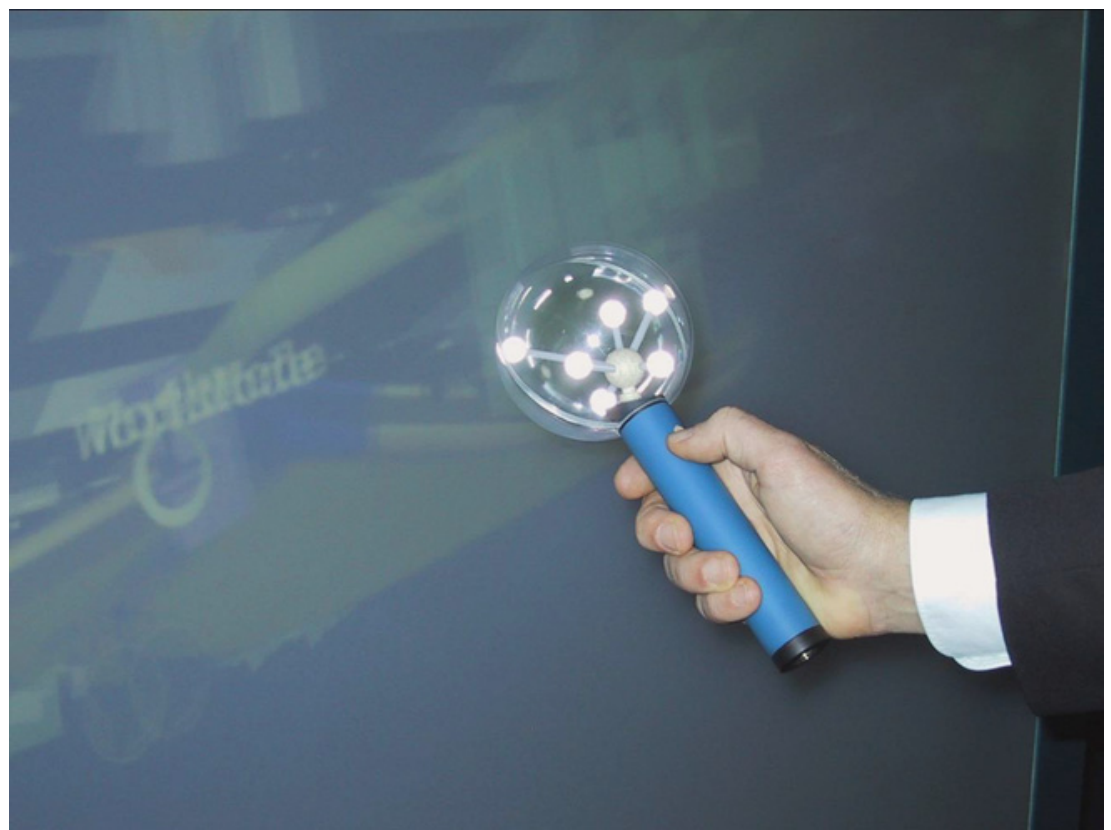

Fig. 6. An optically tracked wireless device with 6 DOF. 
development of an interaction device, which has virtually no training curve and disappears to the user subjectively when used. In order to increase efficiency, usability, and performance and the sense of presence of the patients, the cognitive information processing when interacting with the VE should ideally be supported by the interface. This means that a new minimalist and intuitive device needed to be developed.

\section{Development of a Fully Intuitive Interaction Device}

One of the major problems when designing and developing new interaction devices for 3-D environments is the lack of specific best practice guidelines. Reported approaches to 3-D environment design (e.g., Bowman, Johnson, \& Hodges, 2001; Bowman, Kruijff, LaViola, Poupyrev, 2001; Eastgate, 2001; Gabbard, Hix, \& Swan, 1999; Kaur, 1998; Wilson, Eastgate, \& D'Cruz, 2002) are difficult to translate into specific guidance for use by developers.

Over the past decade, a number of innovative interaction devices have been proposed, but they are still far from becoming standard tools (Zhai, 1998) and selection may have more to do with cost and availability than usability and comfort. The development of a cheap interaction device, which is intuitive and easy to use, was needed.

New, improved interaction devices can be designed through an understanding of usability problems associated with existing devices. Therefore, a set of prototype devices with different grip requirements were designed in order to investigate preferences (Stefani, Patel, Haselberger, Wiederhold, \& Bullinger, 2005).

\section{Interaction in Virtual Environments}

There are many potential ways for users to interact with a VE, e.g., with speech, gestures, predefined shortcuts, etc.; however, these modes of interaction may place a high memory demand on users who are required to remember these commands/gestures. In applications in which a high level of presence is desired, the input device must not distract the user's attention from the main task. Navigation through a VE should be as natural and intuitive as possible.

Any device should match the perceptual needs of the user (Kalawsky, 1996), take into account the task to be performed and the interrelationship between the task and device from a user's perspective (Jacob, Sibert, McFarlane, \& Mullen, 1994), and adhere to natural mappings in the way it is manipulated, as well as permit movements that coincide with the user's mental model of the type of movement required in a VE (Preece et al., 1994).

\section{Optimization of HCI and Related Input Devices}

A human centered approach to design ensures that developers understand the needs and goals of real users at an early stage in the design process, resulting in a more usable end product. The success of the end product depends on an iterative design and evaluation process. It is important for the design process to allow flexibility and facilitate creativity while also taking into account guidance of technical, task, and user constraints. However, 
there are sometimes difficulties with actually achieving human centered design. The VIEW of the Future-project addressed this by generating a User Requirements Document (URD) within which user companies presented their scenarios-the tasks which they wanted to model in VR, the company's application goals, priorities, and constraints. The requirement for lightweight interaction devices with $6 \mathrm{DOF}$, appropriate to the physical and cognitive ergonomics of the users' hand was not only needed for the specific task of navigation in a virtual maze but was a general requirement of most end-users involved in that project (Volvo, Peugeot, Alenia-Spazio, John Deere).

Well-designed interface tools will not only minimize the users' memory demand but will also improve the quality and productivity of work using new technologies (D'Cruz, Eastgate, Nichols, \& Wilson, 2002).

For our iterative development of interaction devices we first obtained expert feedback on the performance of devices. Then, we conducted Experiment 1 to evaluate the use of the two most preferred interaction devices. The outcomes of this study allowed us to make recommendations concerning the design of future devices, which were reported back to the designers in appropriate form and used to develop a new generation of interaction devices. One of these was evaluated in Experiment 2 to see if the guidance provided to designers resulted in a new device that was more usable.

\section{Testing of the Prototype}

Although often seen as an afterthought in computer interface design, being seen as trivial compared to the cognitive issues, physical ergonomics of devices is in fact critical for successful interaction in VR/VE. Anthropometric measurements of the human hand are essential for the design of handheld devices (Bandera, Kern, \& Solf, 1986), and there are numerous ergonomic grips, which can be afforded by handheld devices by the use of different types of handles, all of which have their advantages (Kapandji, 1992). For the purposes of navigation in VEs, a suitable alternative for a handle has to provide a shape, which allows precise movements, without necessarily transmitting high forces. It is important to consider how the device will naturally be held, and ideally there should be flexibility, i.e., users should be able to hold it in different ways, which could help to avoid fatigue. At the same time, the device has to accommodate the technical equipment required for real-time tracking.

\section{DISCUSSION}

A number of ergonomic and other design issues emerged from the qualitative analysis of Experiment 1, which enabled designers to highlight the problems they would address for a new generation of interaction devices. We aimed to improve upon existing devices by incorporating the good design features of tested devices in the new device, while addressing the negative features and issues emerging from Experiment 1. The basic shape was a combination of the OC (see Fig. 7) and the Mike (see Fig. 8), and subsequently the process of shaping and testing foam models was iterative. 


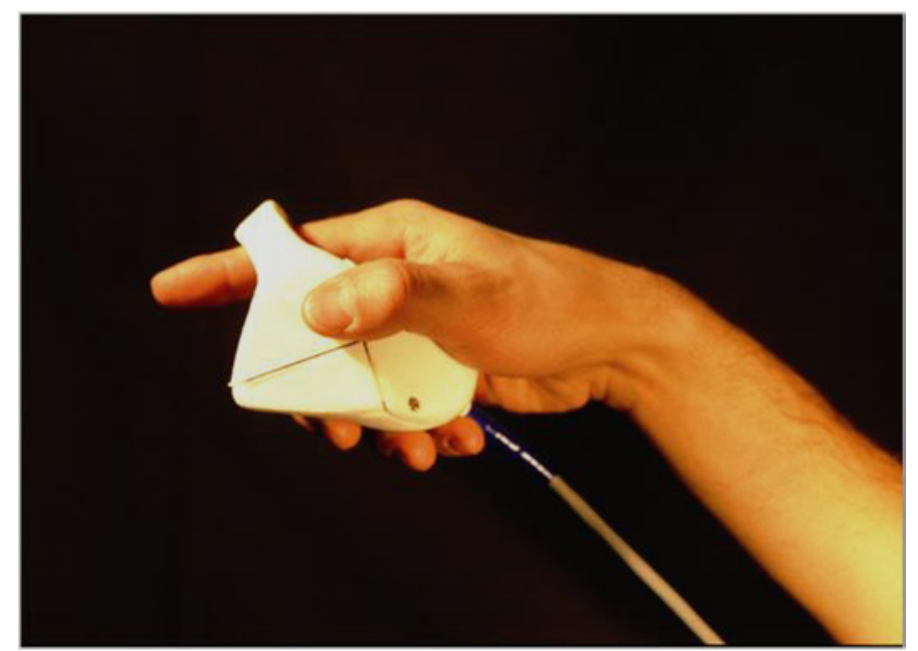

Fig. 7. The OC.

Both visual and haptic sense needs contributed to the final shape of the new device, the Hornet (see Fig. 9).

Experiment 2 was conducted at the VR lab of the Institute of Communication and Computer Systems of the National Technical University of Athens. It is interesting that participants preferred the standing position while using both devices. One disadvantage of the Mike was that none of the participants understood intuitively how to hold it for the first time, for example, it was common for participants to hold the Mike upside down. Participants found it difficult to estimate when the Hornet's pointer was parallel with the floor because, unlike the Mike, it lacked flat surfaces, which provide cues on the direction of the pointer.

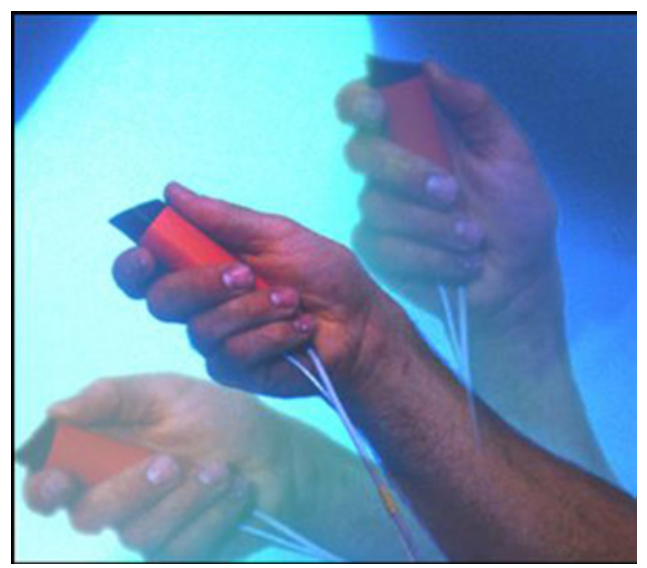

Fig. 8. The Mike. 


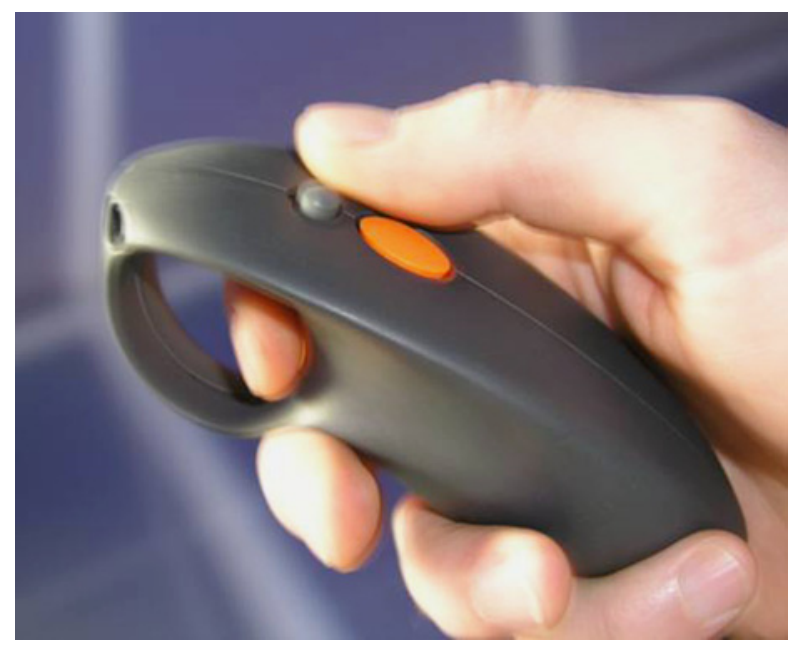

Fig. 9. The Hornet.

Another point to mention is that participants found it difficult to use the Hornet while in a seated position. Although most VR systems are designed to be used in a standing position, issue was addressed in the shape and design of the "dragonfly" as well.

The dragonfly is a wireless, low-cost, and lightweight pointing interaction device for real-time optical tracking systems. It was developed at the same time as the Hornet by taking into account the findings from the current evaluation work. The concept, technical features, and shape of the devices is different to magnetically tracked devices. Optical tracking provides new possibilities for interacting with VEs, for example, by using lightweight, wireless devices. It has been specifically designed to give the user the option of a greater variety of ergonomic grips, independent of a standing or sitting posture in front of a projection screen. Depending on the projection setup and the task it is possible to change between many different grips to prevent fatigue of the hand (Fig. 10).

The dragonfly (shown in Fig. 11) is wireless to limit interference with the user (see Stefani, Hoffmann, \& Rauschenbach, 2003 for a detailed description of how the dragonfly was designed).

\section{CONCLUSION}

Interaction devices designed for use in VEs need to perform technically as well as be ergonomic and easy to use in order to be accepted by end users. User acceptance of the device is important in its perceived usability, and for effective and efficient operation within a VE. Only interaction devices that can be used intuitively, reliably, and without specific skills can be used with patients suffering from mild to moderate cognitive impairments. Otherwise, performance will go down considerably and might even become worse compared to a matching task in "real reality." In the experiment described here, the dragonfly minimized the cognitive and ergonomic strain when the users had to navigate in the virtual maze and thus raised performance significantly. 


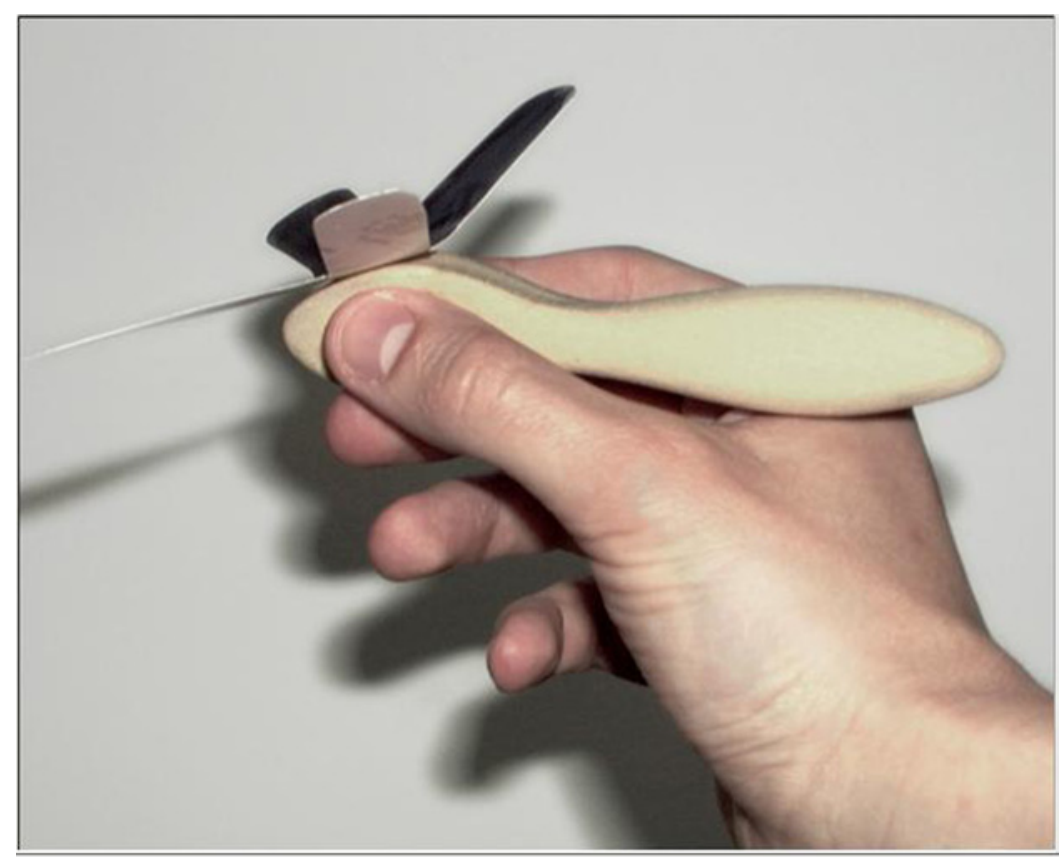

(a)

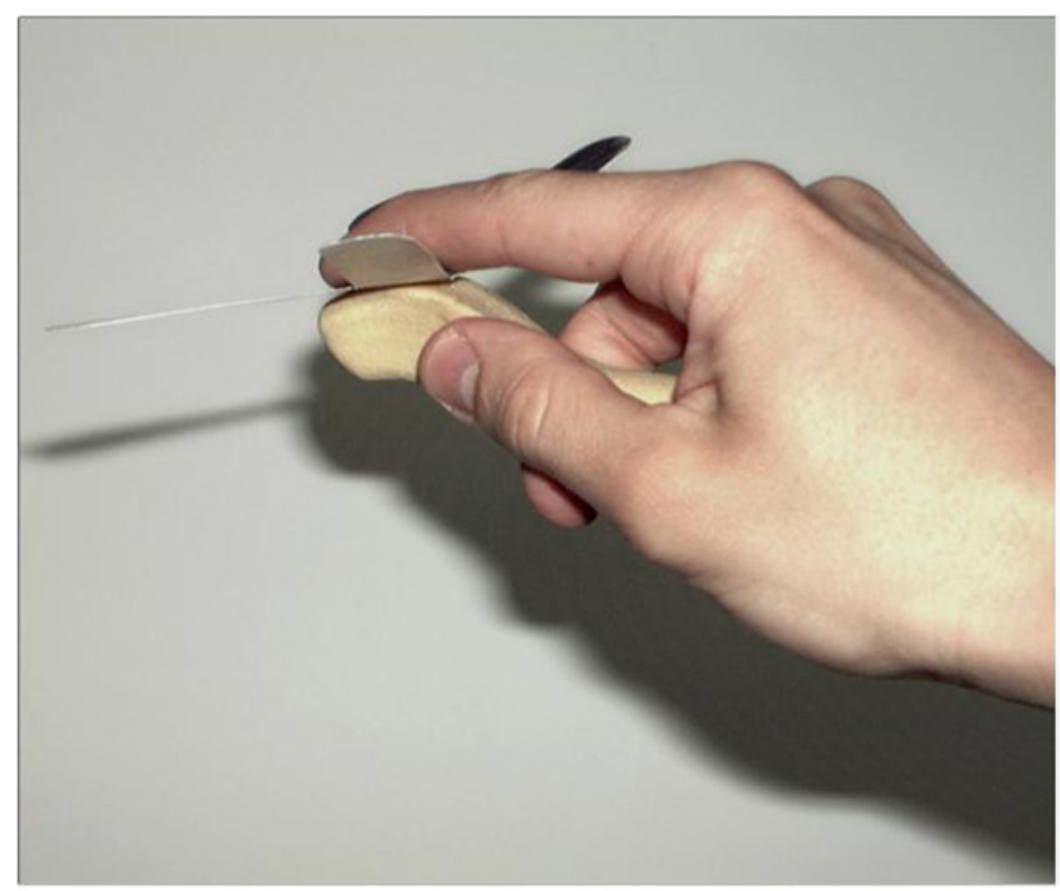

(b)

Fig. 10. Different grip possibilities of the dragonfly. 


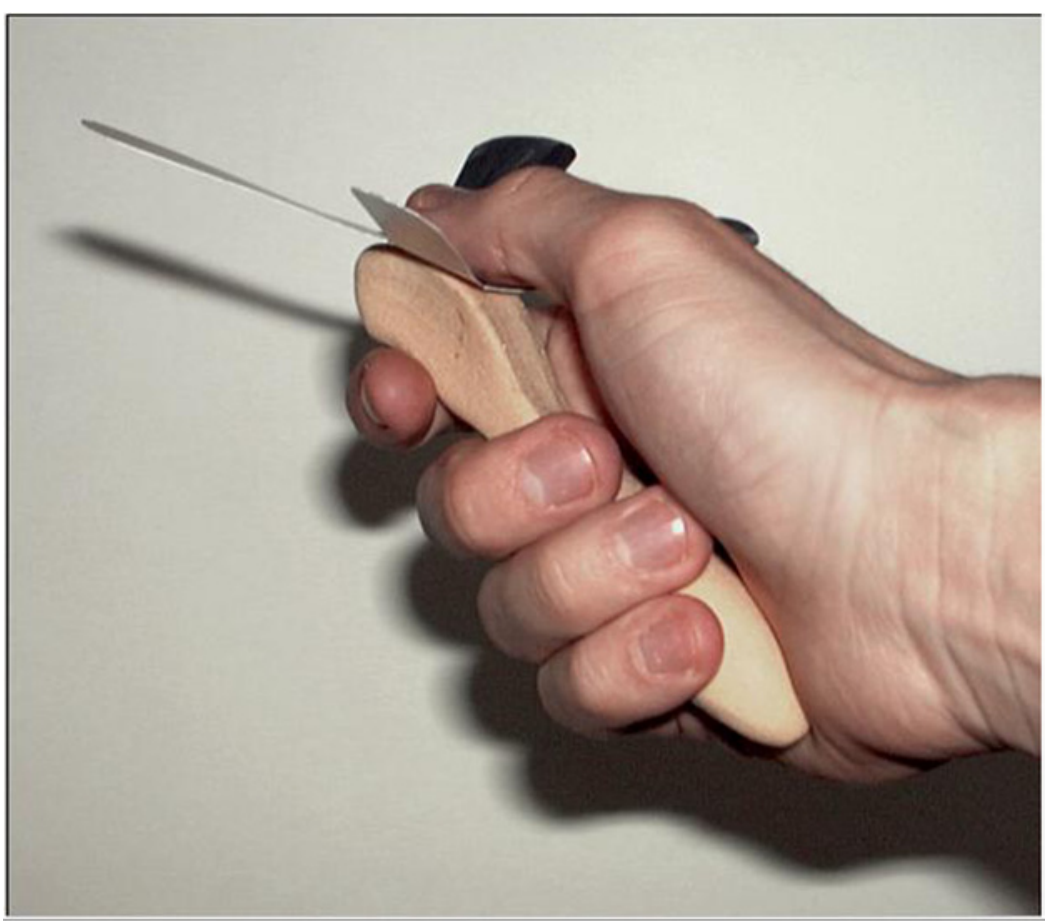

(c)

Fig. 10. Continued.

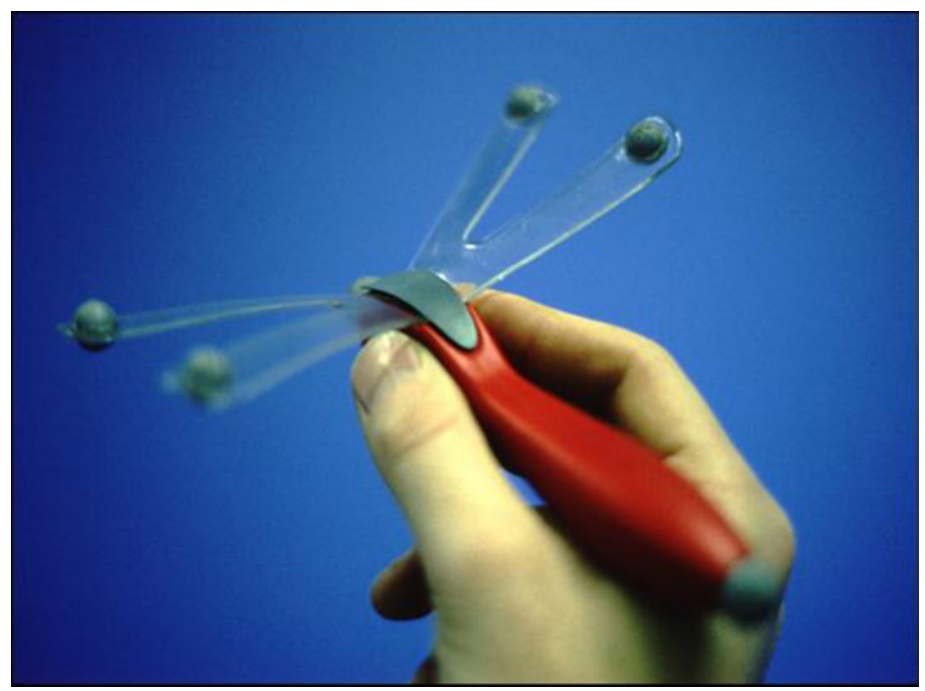

Fig. 11. The dragonfly. 


\section{ACKNOWLEDGMENT}

This work has been funded in part by the EU cofunded project, "VIEW of the Future," contract number IST-2000-26089.

\section{REFERENCES}

Bandera, J. E., Kern, P., \& Solf, J. J. (1986). Leitfaden zur Auswahl, Anordnung und Gestaltung von kraftbetonten Stellteilen. Schriftenreihe der Bundesanstalt für Arbeitsschutz, Dortmund, Fb 494. Bremerhaven: Wirtschaftsverlag NW.

Bowman, D. A., Johnson, D. B., \& Hodges, L. F. (2001). Testbed evaluation of virtual environment interaction techniques. Presence: Teleoperators and Virtual Environments, 10(1), 75-95.

Bowman, D., Kruijff, E., LaViola, J., \& Poupyrev, I. (2001). An introduction to 3-D user interface design. Presence: Teleoperators and Virtual Environments, 10(1), 96-108.

D’Cruz, M., Eastgate, R., Nichols, S., \& Wilson, J. (2002). Human factors challenges. Deliverable for the EU, IST Project Future_Workspaces, IST-2001-38346.

Eastgate, R. (2001). The structured development of virtual environments: Enhancing functionality and interactivity. Unpublished PhD thesis, University of Nottingham, Nottingham, UK.

Gabbard, J., Hix, D., \& Swan, E. (1999). User-centred design and evaluation of virtual environments. IEE Computer Graphics and Applications, 19, 51-59.

Häfner, U. (2002). Entwicklung eines kabellosen Eingabesystems für immersive Umgebungen, PhD thesis, JostJetter Verlag, Heimsheim.

Jacob, R., Sibert, L., McFarlane, D., \& Mullen, P., Jr. (1994). Integrality and separability of input devices. ACM Transactions on Computer-Human Interaction, 1(1), 3-26.

Kalawsky, R. (1996). Exploiting virtual reality techniques in education and training: Technological issues. Prepared for AGOCG. Advanced VR Research Center, Loughborough University of Technology. SIMA Report Series, ISSN 1356-5370.

Kapandji, I. A. (1992). Funktionelle Anatomie der Gelenke (2nd ed.), Bd. 1: Obere Extremitäten. Stuttgart, Germany: Ferdinand Enke Verlag.

Kaur, K. (1998). Designing virtual environments for usability, PhD thesis, City University London, UK.

Mine, M. (1995). Virtual environment interaction techniques (Technical Report TR95-018): UNC Chapel Hill CS Dept, 1995

Nelson, H. (1976). A modified card sorting response sensitive to frontal lobe defects. Cortex, 12, 313-324.

Poupyrev, I., Billinghurst, M., Weghorst, S., \& Ichikawa, T. (1996). The Go-Go interaction technique: Nonlinear mapping for direct manipulation in VR. In Proceedings of the ACM Symposium on User Interface Software and Technology, New York, USA, S. 79-80.

Preece, J., Rogers, Y., Sharp, H., Benyon, D., Holland, S., \& Carey, T. (1994). Human-Computer Interaction. Wokingham, UK: Addison-Wesley.

Roessler, A. (2001). Ein System für die Entwicklung von räumlichen Benutzungsschnittstellen. (IPA-IAO Forschung und Praxis; 334). Zugl.: Stuttgart, University, Dissertation, 2001. Jost-Jetter; ISBN:3-93138859-X.

Stefani, O., Hoffmann, H., \& Rauschenbach, J. (2003). Design of interaction devices for optical tracking in immersive environments. In Proceedings of the HCII2003, June 22-27, 2003, Crete, Greece.

Stefani, O., Patel, H., Haselberger, F., Wiederhold, B., \& Bullinger, A. (2005). Developing interaction devices with 6 DOF for virtual environments. In Proceedings of the HCII2005, Las Vegas, USA.

Wilson, J. R., Eastgate, R. M., \& D'Cruz, M. (2002). Structured development of virtual environments. In K. Stanney (Ed.), Handbook of Virtual Environments (pp. 353-378). Mahwah, NJ: Erlbaum.

Zhai, S. (1998). User performance in relation to 3D input device design. ACM Computer Graphics, 32(4), 50-54. 